\title{
Modeling the Speed Dynamics of a Car
}

\author{
Vlad Mureşan, Mihail Abrudean, Valentin Sita, Iulia Clitan, Mihaela-Ligia Ungureşan, and Roxana \\ Carmen Cordoş
}

\begin{abstract}
In this paper, we present two solutions for modeling the speed dynamics of a car, based on a linear and nonlinear model. The two models are founded on experimental data obtained from a real car. The process time constants were computed using an original algorithm. The accuracy of the two proposed mathematical models is proved through simulation and through the small values obtained for the considered quality indicator. The nonlinear model is proposed to improve the results obtained in the case of the linear model. Also, the model is designed considering the gear.
\end{abstract}

Index Terms - Linear model, nonlinear model, experimental data, speed dynamics, mathematical modeling, simulation.

\section{INTRODUCTION}

The main aim of the paper is to determine an appropriate model for the speed dynamics of a car [1]. In this context, we propose two mathematical models: a linear and a nonlinear model [2], [3]. Both the models are determined based on the processing of experimental data.

For acquiring data parameters from a car, we used specific serial or USB to CAN converters. The parameters from this paper were obtained using an OBD2 Diagnostic Scanner, named VAG-COM 409.1., produced by the company Ross-Tech, LLC/Uwe M. Ross. For using the diagnostic scanner, an application must be installed, as well as an USB driver. In this case, the application suitable for the VAG-COM 409.1. OBD2 Diagnostic Scanner is the VCDS software that contains a user-friendly interface, where one can easily find all the car parameters in specific menus and submenus. The program options from the VCDS release 18.2.1 are presented in Fig. 1, where the OBD2 Diagnostic Scanner port can be selected.

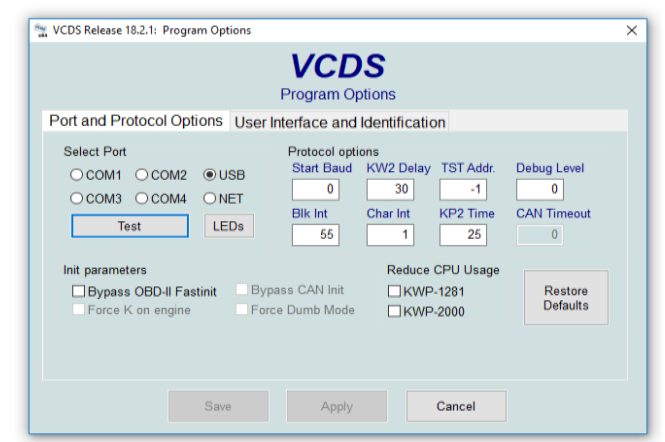

Fig. 1. Program options from the VCDS application, release 18.2.1.

Manuscript received October 20, 2018; revised December 12, 2018.

The authors are with Technical University of Cluj-Napoca, Cluj-Napoca, 400114, Romania_e-mail:_Vlad.Muresan@aut.utcluj.ro, Mihail.Abrudean@aut.utcluj.ro, Valentin.Sita@aut.utcluj.ro, Iulia.Inoan@aut.utcluj.ro, Mihaela.Ungureşan@chem.utcluj.ro, Roxana.Cordos@mis.utcluj.ro,
The concept of the application is simple: all the parameters are uploaded in real time, and each of them has a checkbox. The data can be logged to a .CSV file, but only the parameters with checked checkboxes are saved. The buttons and their functions in the VCDS application are presented in Table 1. For our analysis, we need the following parameters: Engine Speed - (G28), Engine Load, Vehicle Speed, Fuel Consumption - Signal, Fuel Consumption - Equivalent, Acceleration Pedal Position - Sensor 1 (G79).

TABLE I: BUTTONS AND FUNCTIONS IN THE VCDS APPLICATION

\begin{tabular}{|c|c|c|c|c|}
\hline Button & Turbo & $\log$ & Stop & $\begin{array}{l}\text { Done, } \\
\text { Close }\end{array}$ \\
\hline Function & $\begin{array}{l}\text { Increase } \\
\text { the } \\
\text { sample } \\
\text { rate }\end{array}$ & $\begin{array}{l}\text { Start } \\
\text { loggi } \\
\text { ng } \\
\text { data }\end{array}$ & $\begin{array}{l}\text { Finish } \\
\text { logging } \\
\text { specific } \\
\text { data }\end{array}$ & $\begin{array}{l}\text { Finish } \\
\text { logging all } \\
\text { the data }\end{array}$ \\
\hline
\end{tabular}

The innovative character of the paper is in the fact that during the parameters acquisition, we used a car with an automatic gear box. Also, we did not use the cruise control option during the experiments. In this manner, we could measure the speed evolution, and all the interconnected parameters, without the influence of a controller.

\section{The Proposed CAR Movement Linear MODEL}

In this paragraph, we present the procedure to determine a linear model for the considered process (the car movement). The proposed model is based on the acceleration pedal position $\mathrm{u}(\mathrm{t})[\%]$ as the main input signal in the process, and the car speed $y(t)[\mathrm{km} / \mathrm{h}]$ as the main output signal from the process (both $(u)$ and $(y)$ being functions depending on time $(t)$ independent variable). Due to the fact that the experimental datasets are available, we have applied experimental identification procedures to determine the process mathematical model.

The experiment made to obtain the experimental data can be briefly described as follows: initially, the car is positioned on an approximately flat road (the entire experiment is made on an approximately flat road), and the engine is started; the cruise control option is disconnected, and the engine runs at idling speed (also, the automatic gear box option is set during the experiment); at the moment when $t=t_{0}=0 \mathrm{~s}$, the driver pushes the acceleration pedal (practically the driver applies the input signal $u(t))$ and tries to provide a variation form for the input signal $u(t)$ as close as it is possible from the variation form of an ideal step-type signal; using the data-acquiring system presented in the first paragraph, the experimental data are obtained as value sets of the both $u(t)$ 
is represented by the fact that the two acquired signals $(\mathrm{u}(\mathrm{t})$ and $y(t))$ do not respect the same sampling intervals. The solution for solving this problem is to approximate the variation forms of the two signals using Spline functions, and after that, to simulate the obtained Spline approximations on the same time interval with the same sampling time. In this paper, we have considered in modeling procedure (as case study) only the car speed increasing regime, on the domain $[0 ; 55] \mathrm{km} / \mathrm{h}$. Initially, on this speed increasing interval, 34 experimental data, for each measured signal (both $\mathrm{u}(\mathrm{t})$ and $y(t)$ ), were acquired (on the time period [0;46.7] s), but as it was previously mentioned, the two resulted curves have different sampling intervals. Consequently, using the MATLAB options, for each from the two signals, a Spline approximating function is generated, and after that, the approximating functions are simulated using the same time vector [0:0.1:46.7] $\mathrm{s}$ (the considered sampling time for both functions is $\Delta \mathrm{t}=0.1 \mathrm{~s}$, resulting 468 pair of input - output experimental data). The two experimental curves, after their synchronization through the usage of the Spline functions, are presented in Fig. 2.

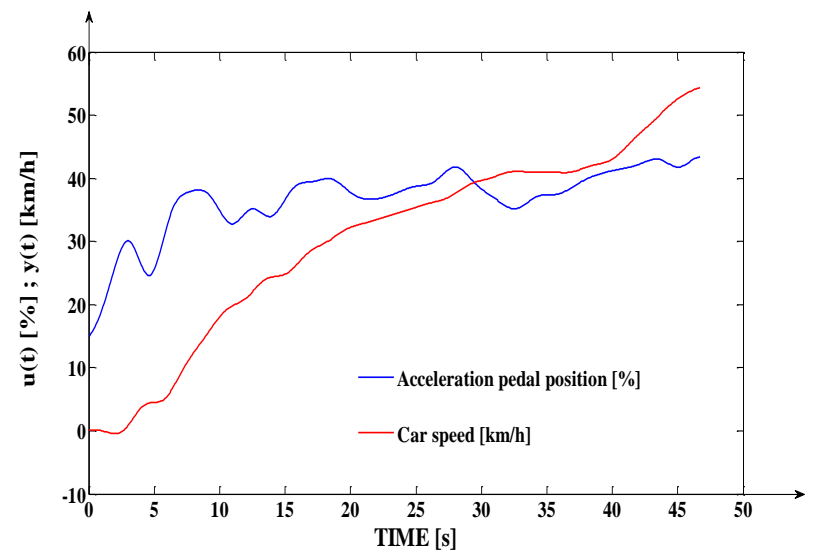

Fig. 2. The experimental curves associated to the $\mathrm{u}(\mathrm{t})$ and $\mathrm{y}(\mathrm{t})$ signals.

As it can be remarked from Fig. 2, the input signal could not be maintained with high accuracy very close from the ideal step type signal variation form. Consequently, the curve associated to the $y(t)$ signal does not have the ideal form of the response of a process with the order higher than I. The initial value of the $\mathrm{u}(\mathrm{t})$ signal, before of selecting the automatic gear box option, was $\mathrm{u}(\mathrm{t})=14.88 \%$. Also, the small decrease of the $y(t)$ signal under the value of $0 \mathrm{~km} / \mathrm{h}$, immediately after the car starting, is due to a local imperfection of the road flatness near the car initial position.

In order to apply an experimental identification procedure, the most simple approach is to use a standard variation form of the input signal. In this case, due to the fact that the driver tried to respect a step type variation form for the $\mathrm{u}(\mathrm{t})$ signal, a solution to the presented problem is to approximate the blue curve variation from Fig. 2 with a step type variation. The most probable step type input signal, which approximates the experimental input signal, is presented in Fig. 3 with blue line. Also, the experimental input signal is figured on Fig. 3 with green line. From Fig. 3, it can be remarked that the most probable step type input signal is very close as variation form from the ideal form (it has a very fast variation (faster than $1 \mathrm{~s}$ ) from the initial value $\mathrm{u}_{0}=14.88 \%$ to the steady state value $\mathrm{u}_{\mathrm{st}}=37.41 \%$ ). The steady state value $\mathrm{u}_{\mathrm{st}}$ is computed by using the relevant section, for this regime, of the experimental curve. The relevant section is figured with red curve on Fig. 3.

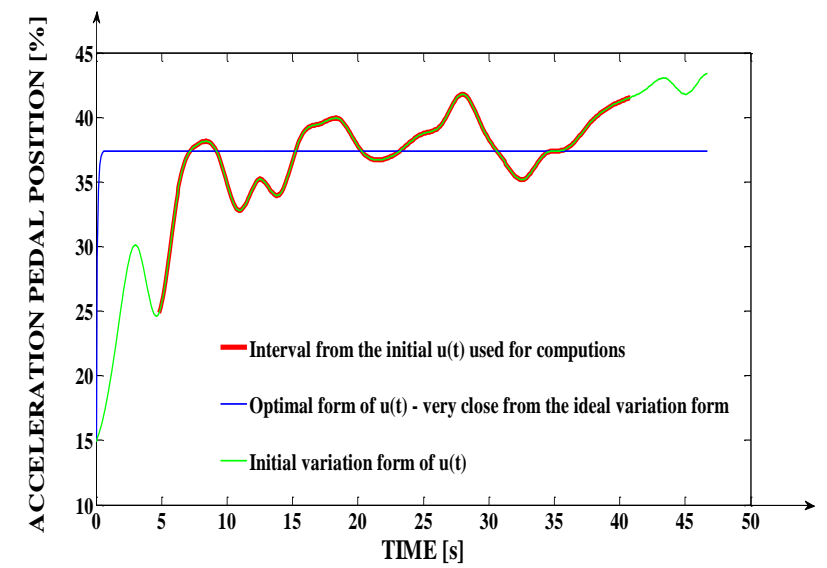

Fig. 3. The computation of the theoretical step type input signal using in the identification procedure.

The relevant section of the experimental input signal, which has to be considered in order to compute the steady state value of the most probable step type input signal results by neglecting the domain associated to the first $5 \mathrm{~s}$ from the $\mathrm{u}(\mathrm{t})$ application (this domain being associated to the most probable step type input signal initial variation, from $\mathrm{u}_{0}$ to $\mathrm{u}_{\mathrm{st}}$ ), and, also, by neglecting the domain associated to the last $6 \mathrm{~s}$ of the $\mathrm{u}(\mathrm{t})$ variation, in which it has a consistent increasing variation (variation which implies the end of the previously set steady state regime of $\mathrm{u}(\mathrm{t})$ ). Using the samples associated to the red curve from Fig. 3 and making their average, the value $\mathrm{u}_{\mathrm{st}}=37.41 \%$ results. Also, due to the fact that the initial value $\mathrm{u}_{0}$ has a relatively small value (it having only the purpose to maintain a small over-speed of the engine relatively to the idling speed), it is not considered in the following computations (practically, in the following computations, we have considered $\mathrm{u}_{0}=0 \%$ ).

From Fig. 2, it can be remarked that the output signal (the $y(t)$ signal) presents an inflection point, this aspect signifying that the treated technical process has the order higher than I. In this context, we propose a mathematical model of second order for the car movement, taking into account that, generally, a second order model can approximate with a relative high accuracy, a model with the order higher than II. Also, our purpose is to find the most simple form of the mathematical model, in order to use it with simplicity in more complex automatic control applications. Consequently, the transfer function of the proposed second order linear mathematical model has the following form:

$$
H_{M}(s)=\frac{Y(s)}{U(s)}=\frac{K_{y}}{T_{1} \cdot T_{2} \cdot s^{2}+\left(T_{1}+T_{2}\right) \cdot s+1}
$$

where $\mathrm{H}_{\mathrm{M}}(\mathrm{s})$ represents the model transfer function, $\mathrm{Y}(\mathrm{s})$ represents the Laplace transformation of the output signal $\mathrm{y}(\mathrm{t}), \mathrm{U}(\mathrm{s})$ represents the Laplace transformation of the input signal $\mathrm{u}(\mathrm{t}), \quad \mathrm{K}_{\mathrm{y}}[\mathrm{km} /(\mathrm{h} \cdot \%)]$ represents the process proportionality constant, respectively $\mathrm{T}_{1}$ and $\mathrm{T}_{2}$ (both expressed in seconds [s]) represent the process time constants. The process proportionality constant is given by the equation:

$$
K_{y}=\frac{y_{s t}-y_{0}}{u_{s t}-u_{0}},
$$


constant, respectively, and $T_{1}$ and $T_{2}$ (both expressed in seconds [s]) represent the process time constants. The process proportionality constant is given by the equation:

$$
K_{y}=\frac{y_{s t}-y_{0}}{u_{s t}-u_{0}}
$$

where the values $u_{0}=0 \%$ and $u_{s \mathrm{t}}=37.41 \%$ were previously introduced in the paper, respectively, $y_{0}=0 \mathrm{~km} / \mathrm{h}$ represents the initial value of the output signal, and $y_{s t}=44.04 \mathrm{~km} / \mathrm{h}$ represents the steady-state value of the output signal. The values of $y_{0}$ and $y_{s t}$ result from the experimental output signal presented in Fig. 2. In the case of $y_{0}$ value, we have considered the small decrease of the output signal under the value of $0 \mathrm{~km} / \mathrm{h}$ as insignificant. Also, the value of $y_{s t}$ is considered the last value of $y(t)$, measured before the moment $t=40.6 \mathrm{~s}$, when its value starts to increase rapidly. The values domain associated with the fast increase of the output signal from the right part of the graph is considered irrelevant for the $\mathrm{K}_{\mathrm{y}}$ proportionality constant computation. After applying (2), the value $K_{y}=1.177[\mathrm{~km} /(\mathrm{h} \cdot \%)]$ is obtained.

The procedure for determining the values of the two time constants $T_{1}$ and $T_{2}$ is based on an iterative algorithm. The following are the algorithm stages:

1) Taking into account the process settling time (considered $t_{s t}=40.6 \mathrm{~s}$, which corresponds to the last value of $y(t)$ considered for $K_{y}$ computation), the two time constants $T_{1}$ and $T_{2}$ are initialized at the values $2 \mathrm{~s}$ and $6 \mathrm{~s}$, respectively (these values are chosen due to the fact that their sum is $8 \mathrm{~s}$, a value that represents approximately the fifth part of $t_{s t}$, a ratio that can be frequently obtained in practice; also the initial ratio of 3 between the values of the two time constants was imposed as the initial search parameter);

2) As a quality indicator for the proposed model accuracy, the mean square error (MSE) was chosen, computed between the experimental output signal and the proposed model response obtained through its simulation [4];

3) The time constants modification step is set to value $\Delta T=$ $0.1 \mathrm{~s}$, a step considered small enough to provide a high accuracy of the determined model;

4) At each iteration, the values $T_{1}$ and $T_{2}$ increase with the value of $\Delta \mathrm{T}$, and after that, the proposed model is simulated at the most probable step input signal, resulting, after the simulation, in the MSE value between the experimental response and the simulated one;

5) Stage 4 is repeated, until the MSE value starts to increase from an iteration to the next one; the obtained values of the time constants, at the previous iteration, are considered to be intermediary solutions;

6) The value of $T_{1}$ obtained at 5 decreases with the value of $\Delta \mathrm{T}$ at each iteration, and the MSE value is tested;

7) Stage 6 is repeated, until the MSE value starts to increase from an iteration to the next one; the obtained value of $T_{1}$, at the previous iteration, is considered to be the final solution;

8) The value of $T_{2}$ is fine-tuned (with multiples of $\Delta T$ ) near the value obtained at Stage 5, until the resulting MSE has the best value that can be obtained - the value of $T_{2}$ obtained at this stage is considered the final solution.

After applying the previously presented stages, the values of $T_{1}=5 \mathrm{~s}$ and $T_{2}=10.5 \mathrm{~s}$ are obtained. Considering these values, and also the value determined for $K_{y}$, the comparative graph between the experimental response and the model response at the most probable input step-type signal, is presented in Fig. 4. In Fig. 4, the proposed model response at the experimental input signal (from Fig. 2) is presented, too. From Fig. 4, it can be seen that the green curve is superposed over the blue curve with a higher accuracy than the red curve (more precisely, the proposed model gives better results for the most probable input step-type signal, due to the fact that it was determined using this signal).

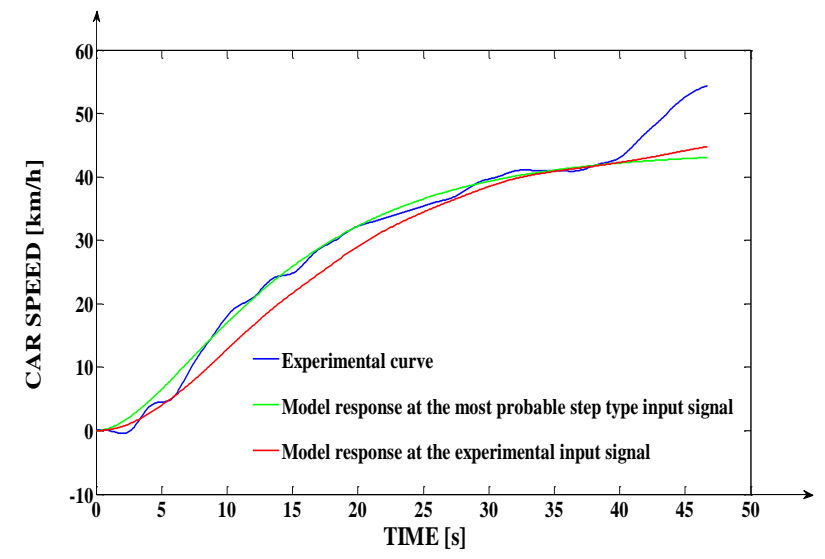

Fig. 4. Simulation of the primary form of the proposed linear model.

This aspect is proved, also, by the values of the two computed errors $\left(\mathrm{MSE}_{1}<\mathrm{MSE}_{2}\right): \mathrm{MSE}_{1}=2.96 \mathrm{~km} / \mathrm{h}$ (between the green and the blue curves) and $\mathrm{MSE}_{2}=3.34$ $\mathrm{km} / \mathrm{h}$ (between the red and the blue curves). The two errors are computed, each, on 468 pairs of samples. Considering the range of values of the output signal $y(t)([0 ; 55] \mathrm{km} / \mathrm{h})$, the two errors are very small, proving the high validity of the proposed linear model. However, we have considered in the two errors computation the values domain associated with the fast increase of the output signal from the right part of the graph, the fact that increases consistently their values. The main conclusion that results from Fig. 4 is that we have to adjust the proposed model time constants to minimize the error between the experimental curve and the model response at the real (experimental) input signal (to obtain a precise model in relation to the real process). Considering this conclusion, if we apply the previously presented methodology to determine the values of $T_{1}$ and $T_{2}$, but if we consider the experimental input signal in the algorithm, the values $T_{1}=5 \mathrm{~s}$ and $T_{2}=9 \mathrm{~s}$ are obtained. Using these values, we obtain the final form of the proposed model. In Fig. 5, the same simulations as in Fig. 4 are presented, but now they take the new values $T_{1}$ and $T_{2}$ into account.

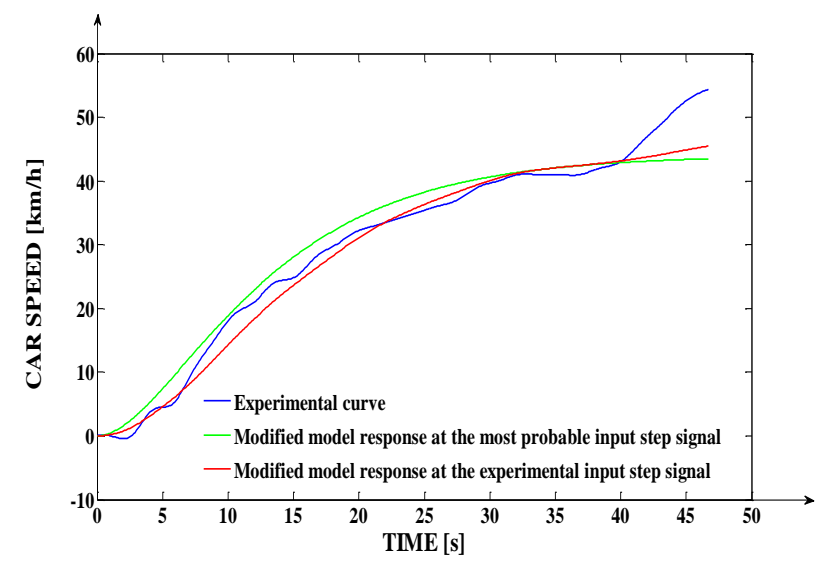

Fig. 5. Simulation of the final form of the proposed linear model. 
From Fig. 5, the consistent improvement of the results, in the case when the experimental input signal is applied, can be observed. This aspect is proved, also, by the error values (computed on the 468 pairs of samples), consequently $\mathrm{MSE}_{3}$ $=3.27 \mathrm{~km} / \mathrm{h}>\mathrm{MSE}_{4}=2.60 \mathrm{~km} / \mathrm{h}\left(\mathrm{MSE}_{3}\right.$ is computed between the green and the blue curves, respectively, and $\mathrm{MSE}_{3}$ is computed between the red and the blue curves). The effect of the values domain associated with the fast increase of the output signal from the right part of the graph also occurs in this case.

\section{The Proposed CAR Movement Nonlinear Model}

In the previous paragraph, an accurate linear mathematical model was proposed for the car speed dynamics. In this paragraph, a nonlinear mathematical model is proposed, with the purpose to obtain an even better accuracy. A solution in this context is to consider in the model structure, the gear, signal, which is also acquired using the procedure described in the first paragraph. Practically, the gear will be the second input signal in the process, and it is notated with $u_{1}(t)$. The experimental evolutions, in relation to time $(t)$, of the two input signals in the process, are presented in Fig. 6.

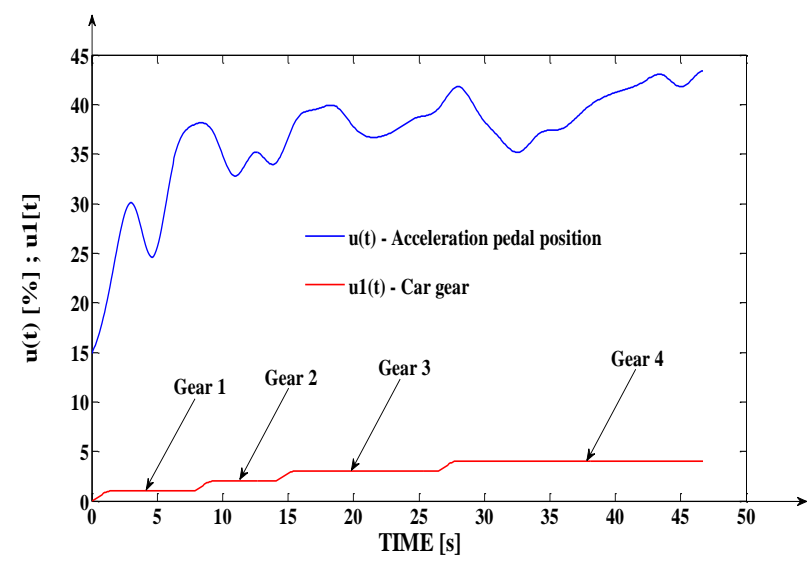

Fig. 6. Evolutions of the two input signals, in relation to time $(t)$.

From Fig. 6, it results that the gear changes automatically (consecutively from 1 to 4 ) due to the maintaining of the $u(t)$ signal. Practically, the gear change depends on the engine speed (which is a measured signal, too), but in this application, the connection between the engine speed and the gear is not necessary to be made because we have an access to the data generated by the acquisition system. In future applications, in which we will implement an automatic control system [5]-[7] for the car speed control, a solution for the automatic gear selection, in relation to the engine speed, will have to be studied. Also, from Fig. 6, it can be remarked that we avoid the discontinuities due to the gear change, by using fast linear increasing variations to connect the consecutive gears. This mathematical adjustment is made to treat the process as a nonlinear one without having discontinuities, and it does not influence significantly the process work. Practically, we made the mentioned mathematical adjustment to propose a unitary nonlinear model, which is more than a viable alternative to the hybrid model.

The most simple and efficient method to introduce the effect of $u_{1}(t)$ input signal in the process mathematical model is to consider the process proportionality constant $K_{y}$ as a function depending on $u_{1}(t)\left(K_{y}=K_{y}\left(u_{1}(t)\right)\right)$ signal. In this context, we define the function $\mathrm{u}_{1}(\mathrm{t})$, as the following:

$$
u_{1}(t)=\left[\begin{array}{llll}
g_{1}(t) & \mathrm{g}_{2}(t) & g_{3}(t) & \mathrm{g}_{4}(t)
\end{array}\right],
$$

where the $g \ldots(t)$ functions are defined by

$$
g_{i}(t)=\left\{\begin{array}{l}
0, \text { if the gear is different than } i \\
1, \text { if the gear is equal to } i
\end{array},\right.
$$

and $i \in\{1 ; 2 ; 3 ; 4\}$.

Also, we consider the adjusted value of the process proportionality consistent, being $K_{y 1}(t)=K_{y}(t)-1$. The proposed solution for determining the connection between the $K_{y}(t)$ and $u_{1}(t)$ functions is to use a column-weighting vector, which, under the transposed form, is

$$
A^{\tau}=\left[\begin{array}{llll}
a_{1} & a_{2} & a_{3} & a_{4}
\end{array}\right],
$$

where the $a_{i}$ coefficients, for $i \in\{1 ; 2 ; 3 ; 4\}$, are constant, and the notation $\tau$ signifies the transposed form of the weighting vector A. Consequently, the process proportionality constant is given by the $K_{y}(t)=K_{y 1}(t)+u_{1}(t) \cdot \mathrm{A}$, equation, which, after making the computations, becomes

$$
K_{y}(t)=K_{y 1}(t)+a_{1} \cdot g_{1}(t)+a_{2} \cdot g_{2}(t)+a_{3} \cdot g_{3}(t)+a_{4} \cdot g_{4}(t) \cdot(6)
$$

The iterative algorithm used to determine the a.... weighting coefficient, follows the stages:

1) All the a.... weighting coefficients are initialized at the value 0.1 .

2) The searching step for the a.... weighting coefficients is set to $\Delta \mathrm{a}=0.05$.

3) The variable $\mathrm{j}$ is initialized to the value 1 .

4) At each iteration, the value of $a_{j}$ increases with $\Delta \mathrm{a}$, and the proposed model is simulated at the experimental input signal, resulting in the corresponding MSE between the experimental and the simulated response.

5) Stage 4 is repeated, until the MSE value start to increase from an iteration to the next one; the obtained value of $a_{j}$, at the previous iteration, is considered as final solution for the weighting coefficient.

6) The variable $j$ is increased with the value 1 , and stages 4 and 5 , respectively, are repeated.

7) The algorithm is stopped when $j=5$ (at this moment, the values of the weighting coefficients $a_{1}, a_{2}, a_{3}$, and $\mathrm{a}_{4}$ are determined).

After applying the previously presented algorithm, the following values are obtained for the a.... coefficients: $a_{1}=1$; $a_{2}=1.25 ; a_{3}=0.9$ and $a_{4}=1$. An important remark is that the commutation of the $K_{y}(t)$ from value to another one is made following fast linear variations compensate the fast increase of the output signal from the right part of Figs. 2, 4, and 5, the $\mathrm{a}_{4}$ initially obtained form is adjusted, resulting in a variable one. The proposed form for the $a_{4}(t)$ coefficient, is

$$
a_{4}(t)=a_{4}+\left(1-\frac{T_{11}}{T_{11}-T_{22}} \cdot e^{-\frac{t}{T_{11}}}-\frac{T_{22}}{T_{22}-T_{11}} \cdot e^{-\frac{t}{T_{22}}}\right) \cdot a_{5}\left(t-\tau_{1}\right)
$$

where the time constants $T_{11}=0.5 \mathrm{~s}$ and $T_{22}=0.6 \mathrm{~s}$ model $\mathrm{a}$ 
fast variation of the $a_{4}(t)$ variable coefficient from a value to another one, respectively, and $a_{5}\left(t-\tau_{1}\right)$ represents a step-type signal. The steady-state value of $a_{5}\left(t-\tau_{1}\right)$ is $a_{5 \mathrm{st}}=0.65$, which is iteratively determined in order to obtain the lowest value of MSE between the experimental and the simulated responses (obviously, the precision used for the value of $a_{5}$ is 0.05 ). The value of the delay $\tau_{1}$ is set to $12 \mathrm{~s}$, to control the commutation of $a_{4}(t)$, only at the moment when the fast increase of the output signal occurs.

Considering the previously proposed solution, the variation, in relation to time, of the $K_{y}\left(u_{1}(t)\right)$ process proportionality constant, is presented in Fig. 7.

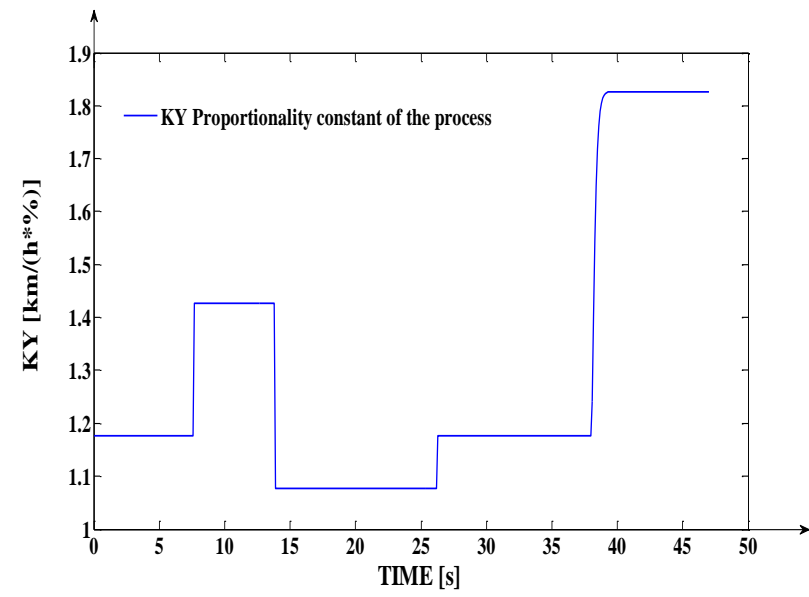

Fig. 7. Proposed variations for the $K_{y}$ process proportionality constant.

Having the $K_{y}\left(u_{1}(t)\right)$ function, the nonlinear model of the process can be simulated. The comparative graph between the experimental response of the process and the response obtained through the simulation of the proposed nonlinear model, at the experimental input signal, is presented in Fig. 8.

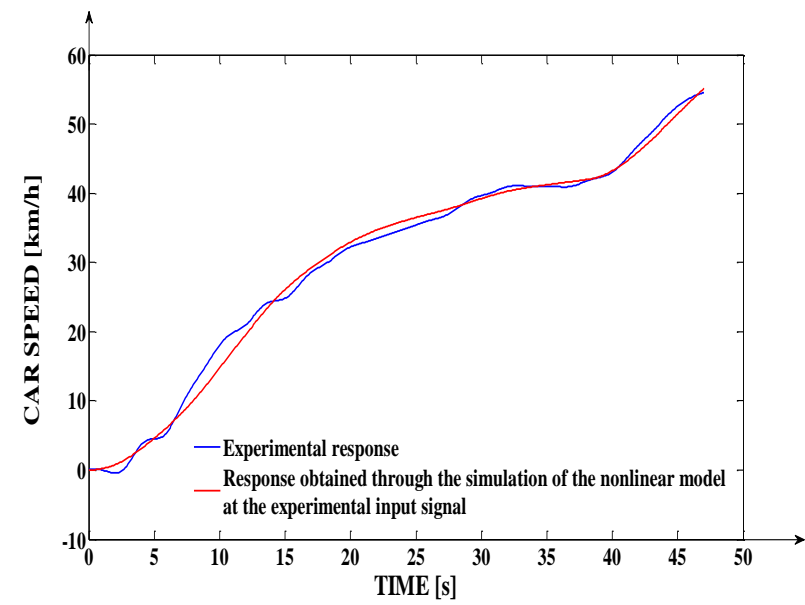

Fig. 8. Comparative graph between the experimental and the simulated response of the process.

From Fig. 8, results a better superposition of the two responses, comparatively with the case of the linear model. This aspect is also proved with a much lower MSE value (than in the case of the linear model): $\mathrm{MSE}_{5}=1.12 \mathrm{~km} / \mathrm{h}$ (computed on 468 pairs of samples as well).

As a conclusion, the nonlinear model presented in this paper section is more accurate than the linear model presented in the previous paper section. However, the lower value of the MSE obtained in the case of the nonlinear model is mainly due to a better superposition of the simulated response over the experimental one, on the domain associated with the response fast increase, from the right part of Fig. 8.

\section{CONCLUSIONS}

In this paper, we present two solutions for modeling of the speed dynamics of a car. In the modeling approach, only the car-speed-increasing regime, on the domain of $[0 ; 55] \mathrm{km} / \mathrm{h}$, is considered. The first proposed model is a linear one, having the advantage of a simpler usage in practice and of a simpler inclusion in an automatic control system, for the speed control. The main disadvantage of the linear model is its lower accuracy. The second proposed model is a nonlinear one, having the main advantage of a higher accuracy. The main disadvantage of the nonlinear model is a more complicated application in practice, the aspect given by a more complex implementation algorithm. The nonlinear model is also more difficult to simulate because a variation form of the car gear (in relation to time) has to be predefined for its simulation. In practice, the use of the nonlinear model has to be associated with an automatic algorithm for determining the gear, in relation to the car engine speed.

The procedures used to determine the structure parameters of both linear and nonlinear models represent original elements. Also, both models are determined by processing experimental data that were acquired using a real car.

Future research will be focused on proposing mathematical models in a speed-decreasing regime as well. Also, the proposed models will be included in automatic control structures for speed control.

\section{ACKNOWLEDGMENTS}

The authors express their gratitude for Prof. Tiberiu Coloşi for his help in the paper elaboration.

\section{REFERENCES}

[1] M. Vollrath, S. Schleicher, and C. Gelau, "The influence of cruise control and adaptive cruise control on driving behaviour - A driving simulator study," Accident Analysis and Prevention, vol. 43, no. 3, pp. 1134-1139, 2011.

[2] V. Mureşan and M. Abrudean, "Temperature modelling and simulation in the furnace with rotary hearth," in Proc. 2010 IEEE AQTR 2010 17th edition, Cluj-Napoca, Romania, pp. 147-152, May 28-30, 2010.

[3] H.-X. Li and C. Qi, Spatio-Temporal Modeling of Nonlinear Distributed Parameter Systems: A Time/Space Separation Based Approach, 1st Edition, Springer, 2011, pp. 10-32

[4] T. Coloşi, M. Abrudean, M.-L. Ungureşan, and V. Mureşan, Numerical Simulation of Distributed Parameter Processes, Springer, 2013 pp.264-280.

[5] J. Love, Process Automation Handbook, Springer, 2007, pp. 250-272.

[6] F. Golnaraghi and B. C. Kuo, Automatic Control Systems, Wiley, 2009, pp. 487-510.

[7] W. S. Levine, The Control Handbook, Second Edition: Control System Applications, Second Edition (Electrical Engineering Handbook), 2010, Publisher: CRC Press, 2 edition.

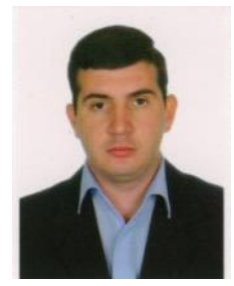

Vlad Mureşan is an associate professor at Technical University of Cluj-Napoca, Automation Department, Romania.

His research interests include mathematical modeling and numerical simulation of distributed parameter processes, industrial plant control, isotope separation processes, advanced control of the metallurgical processes, intelligent control, biomedical processes, etc.

He has 110 papers published in journals or communicated at international conferences, as well as 7 books and 10 research contracts, where he managed or was a member of a research team. 


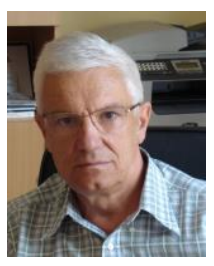

Mihail Ioan Abrudean is a professor at Technical University of Cluj-Napoca, Automation Department, Romania.

His research interests include mathematical modeling and numerical simulation with distributed parameter processes, robust nonlinear control of isotope separation columns, robust-predictive control of isotope separation columns, etc.

$\mathrm{He}$ has 253 papers published in journals or communicated at international conferences, 5 patents, 15 books, and 82 research contracts he managed or was a member of the research team.

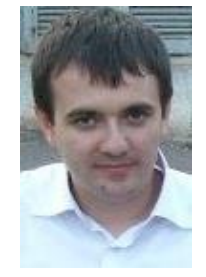

Ioan-Valentin Sita is a lecturer at Technical University of Cluj-Napoca, Automation Department, Romania.

His research interests include building automation, Internet of Things, smart-city, cyber-physical systems, etc.

He has 34 scientific papers, 14 as the first author; 4 papers in magazines (AISC), 2 as the first author; 30 works at international conferences, 12 as the first author (5 ISI Proceedings Conferences); 2 research contracts where he was the manager and 2 where he was a research team member.

$\mathrm{He}$ is the founding member of the scientific partnership between the Technical University of Cluj-Napoca and the KNX Association, and also a SRAIT and IEEE member.

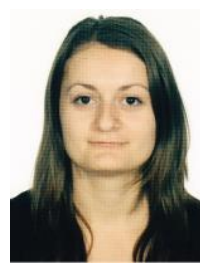

Iulia Clitan graduated from the Automation and Computer Science Faculty and completed her master's degree in advanced process control at the Technical University of Cluj-Napoca, Romania. She received her doctorate in systems engineering from the same university. Her research interests are industrial process control and biomedical engineering. She is currently a lecturer at the Automation Department, the Technical University of Cluj-Napoca.

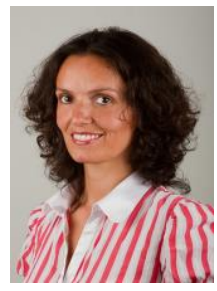

Mihaela-Ligia Unguresan is an associate professor at Technical University of Cluj-Napoca, Physics and Chemistry Department, Romania.

Her research interests include chemical kinetics of rapid reactions, isotopic exchange reactions, modeling and simulation of physical-chemical processes, synthesis of chemical compounds marked with stable isotopes, etc.

She has 118 papers published in journals or communicated at international conferences, as well as

15 books and 30 research contracts where she was the manager or a research team member.

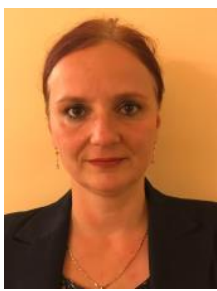

Roxana Carmen Cordoş is a associate professor at Technical University of Cluj-Napoca, Industrial Engineering and Management Department, Romania.

Her research interests include mathematical modeling and numerical simulation with distributed parameter processes, modeling of economical processes, etc. 